\title{
Modeling and parameters estimation of a Spatial Predator-Prey distribution
}

\author{
Denis Ndanguza*1 $^{*}$ Jean Pierre Muhirwa ${ }^{1}$ and Anatholie Uwimana ${ }^{2}$ \\ ${ }^{1}$ Department of Mathematics, College of Science and Technology, \\ University of Rwanda, P.O. Box 3900 Kigali, Rwanda. \\ ${ }^{2}$ African Institute of Mathematics (AIMS) - Rwanda \\ *Principal author email: dndanguza@gmail.com
}

\begin{abstract}
Predator prey interactions are important in ecology and most of time in the analysis, the two antagonists are assumed to be in a closed system. The aim of this study is to model the unclosed predator-prey system. The model is built and simulated data are computed by adding noise on deterministic solution. Therefore, model parameters are estimated using least square method. We compute the two critical points and the stability analysis is carried out and results show that the population is stable at one critical point and unstable at $(0,0)$. The model fits the synthetic data with coefficient of determination $R^{2}=0.9693$ equivalent to $96.93 \%$. Using the residual analysis to test the validity of the model, it is shown that there is no pattern between residuals. To strengthen the validity of the model, the Markov Chain Monte Carlo algorithms are used as an alternative method in parameters estimation. Diagnostics prove the chains' convergence which is the sign of an accurate model. As conclusion, the model is accurate and it can be applied to real data.
\end{abstract}

Key words: predator-prey, spatial distribution, parameters, Metropolis-Hastings algorithm, model diagnostic, stability analysis.

\section{Introduction}

The vast majority of mathematical models which have been used to describe the growth dynamics of populations are based on autonomous equations (Wilson and E. O. 1971). All parameters are constant in time. It is widely recognized that both inherent biological and physical environmental parameters can and in fact often fluctuate in time and that these fluctuations can have significant effects on the dynamics of the populations (Cushing \& Levarge, 2005). In (Jang \& Baglama, 2002), intratrophic predation has been deemed as one of the process of stabilizing the system. This process may have an impact on the system if the predator basic reproductive number becomes bigger than unit; together with the uniqueness of equilibrium from several existing equilibria. Depending on the purpose of the study, one can introduce status in spatial predator prey model. The representative example is found in (Kumar and Kant, 2015), where disease infection can propagate into the system from infected prey or predator. The consequences or the benefits of both migration and diffusion, cross diffusion and limited diffusion of spatial predator-prey models are also widely explained in (Cuddington \& Yodzis, 2000; Liu, 2010). The importance of plugging time independent in spatial predator prey which let both population oscillate in time and space has been investigated in (Lugo \& McKane, 2008).

Some of the population models studied is single species such as Malthusian model where the population increases exponentially without bound when the parameter is positive and decreases 
when the parameter is negative which is not naturally realistic. This Malthusian model has been modified by Verhulst by introducing the carrying capacity. If the population is small, it increases exponentially and bounded by the carrying capacity. This prevents the extinction of a small population. If the population is huge, it decreases to the carrying capacity (Boccara, 2010).

Others consider multi-dimensional predator-prey models for instance direct Lyapunov method has been applied on three-dimensional predator-prey systems whereby classical Lotka-Voltera model of two predators-one prey and vice versa were typically analysed. It was revealed that one species goes to extinction, as a result, the three-dimensional model acts like two dimensional model (Korobeinikov, A. and Wake, 1999).

Furthermore, models for two antagonist species were studied and analysed by different authors using different methods. The behaviour of species in prey-predators is always oscillating because when preys increase, predators get nutrient and their number also increases. Once predators become many, preys decrease because the predation becomes intense i.e. preys are eaten and this causes the shrink of predators. In (Pal, Mahapatra, \& Samanta, 2015), a fractional-ordered predator-prey model was constructed and it was numerically and analytically analysed using two methods, namely, Homotopy Perturbation Method (HPM) and Variational Iteration Method (VIM). The two approaches have shown less computational task with closer numerical and analytical solutions which are quantitatively reliable results. Heterogeneity in spatial predatorprey model has been well explained by (Bergström, Englund, \& Leonardsson, 2006) using Moment Approximation Method. A natural problem to ask then is to know what will happen if there is circulation in an existing boundless homogeneous system containing preys and competitive predators. The aim of this paper is to have a basic understanding of the spatial predator-prey model with conflict between predators due to the competition. To achieve this objective, we explore two methods of parameters estimation. Firstly, the Least Square (LSQ) method is used and secondly, we use a Bayesian approach known as Markov Chain Monte Carlo (MCMC) as novelty in this area of predator-prey models.

Very often authors estimate the model parameters, whereas others prefer to assume them since it is not easy to find data. In our case, we propose the estimation of parameters by using synthetic data. The data used are simulated and fitted to the simple deterministic (discrete-time) proposed predator-prey model. The least-square fit of the model provides estimates for the model parameters (optimal parameters), which are then used to decide on stability analysis. These estimated parameters are further analyzed using MCMC methods. The model solution is achieved by feeding it with a set of simulated data at a given time $(\mathrm{t})$. The value of the variables at any other time can be derived from these data and can be presented in the form of time trends. This paper has four main parts: after the introduction where the literature and background of the topic are given, the spatial model was constructed based on the inflow and out flow of both populations. The numerical simulations of the required models have been done followed by discussions of results and both the LSQ and MCMC methods were used to compute the optimal parameters and to validate the model.

\section{Mathematical formulation of spatial predator-prey model}

To describe the dynamic behavior of predator-prey, we took some simplifications of the model by choosing the following assumptions.

- The preys will grow exponentially up to carrying capacity when the predators are absent. 
- The predator populations will die out in the absence of the prey.

- Predators can consume infinite quantities of prey.

- Emigration of predator population is caused by conflict between them.

- Immigration and reproduction rates are combined in one parameter known as growth rate.

- Environment is homogeneous through time, means both populations are moving randomly through a homogeneous environment.

Therefore, we write down two simultaneous differential equations that represent the dynamism and the interaction between both populations in boundless environment. For our simplicity we just write $N_{1}(t)$ and $N_{2}(t)$ as $N_{1}$ and $N_{2}$, respectively.

$$
\left\{\begin{array}{l}
\frac{d N_{1}}{d t}=\alpha N_{1}\left(1-\frac{N_{1}}{\varepsilon}\right)-d_{1} N_{1} N_{2}-\sigma N_{1} \\
\frac{d N_{2}}{d t}=-d_{2} N_{2}+\beta N_{1} N_{2}-\mu\left(N_{2}\right)^{2}
\end{array}\right.
$$

where variables and parameters are defined in Table 1.

Table 1: Variables and Parameters

\begin{tabular}{|l|l|}
\hline Variables and Parameters & Definition \\
\hline$N_{1}, N_{2}$ & Size of prey and predator populations respectively at time t \\
\hline$\alpha$ & Growth rate of prey \\
\hline$d_{1}$ & $\begin{array}{l}\text { Death rate of prey per meeting of predator because of } \\
\text { predation }\end{array}$ \\
\hline$\varepsilon$ & Carrying capacity constant fixed as 100 \\
\hline$\beta$ & Predator growth rate per prey \\
\hline$\sigma, \mu$ & Emigration rates of prey and predator populations respectively \\
\hline$d_{2}$ & Natural mortality rate of predator \\
\hline
\end{tabular}

\subsection{Estimation of parameters}

Data: Because of mathematical intractability, it is often necessary to investigate the properties of a model using simulated data. In power analysis, simulation refers to the process of generating several thousand random samples that follow a particular distribution. In this paper, we consider $t$ to be $233 \times 1$, and simulated data being $233 \times 2$ since there are two variables and all parameters are fixed. By plugging in the fixed guessed parameters in (1), the new model is therefore

$$
\left\{\begin{array}{l}
\frac{d N_{1}}{d t}=0.801 N_{1}\left(1-\frac{N_{1}}{100}\right)-0: 195 N_{1} N_{2}-0.047 N_{1} ; N_{1}(0)=30 \\
\frac{d N_{2}}{d t}=-0.053 N_{2}+0.1 N_{1} N_{2}-0.0008\left(N_{2}\right)^{2} ; N_{2}(0)=4
\end{array}\right.
$$

The simulation of data is performed using Matlab by solving the system (2) numerically and then add a noise on the solution. Once the data set is found, we minimize the sum of square errors and get estimates summarized in Table 2. 
Table 2: Identification of model parameters by LSQ

\begin{tabular}{llll}
\hline Parameter & Meaning & Initial guess & Estimates \\
\hline$\alpha$ & Growth rate of prey & 0.801 & 0.812 \\
$d_{1}$ & $\begin{array}{l}\text { Death rate of prey } \\
\beta\end{array}$ & 0.195 & 0.201 \\
$\sigma$ & $\begin{array}{l}\text { Predator growth rate } \\
\text { Emigration rates of }\end{array}$ & 0.103 & 0.1001 \\
$\mu$ & $\begin{array}{l}\text { prey } \\
\text { Emigration rates of } \\
\text { predator } \\
\text { Mortality rate of }\end{array}$ & 0.0008 & 0.032 \\
$d_{2}$ & predator & 0.053 & 0.0008 \\
& & & 0.048 \\
\hline
\end{tabular}

\subsection{Stability analysis}

Stability analysis of any system is very important since it shows when and how the system can be controlled over a wide range of time. From the system of equation (1), we have two critical points $\left(N_{1}=0 ; N_{2}=0\right)$ and $\left(N_{1}=\frac{\varepsilon d_{1} d_{2}-\mu \varepsilon(\sigma-\alpha)}{\mu \alpha+\varepsilon \beta d_{1}}, N_{2}=\frac{\beta \varepsilon d_{1} d_{2}-\beta \mu \varepsilon \sigma+\beta \mu \varepsilon \alpha-d_{2} \mu \alpha-d_{1} d_{2} \varepsilon \beta}{\mu^{2} \alpha+d_{1} \mu \varepsilon \beta}\right)$ for which, after substituting estimated values from Table 2 , reads $\left(N_{1}=5 ; N_{2}=3\right)$, meanwhile its stability was plotted and investigated. Linearization of the system around the point $\left(N_{1}=5 ; N_{2}=3\right)$ with Jacobian analysis provided two complex conjugate eigenvalues $q_{1,2}=0.046 \pm 0.55 i$ after substituting the optimal parameters and shape the critical point to be spiral point which is asymptotically stable point. Meanwhile $\left(N_{1}=0 ; N_{2}=0\right)$ provides both negative and positive real eigenvalues $q_{1}=0.59$ and $q_{2}=0.53$, that is shown to be unstable hyperbolic saddle point. Precisely means the extinction of both populations is not possible even though the presence or the absence of predation process, preys are still growing exponentially unless there is no introductory rate of populations in the ecosystem. 


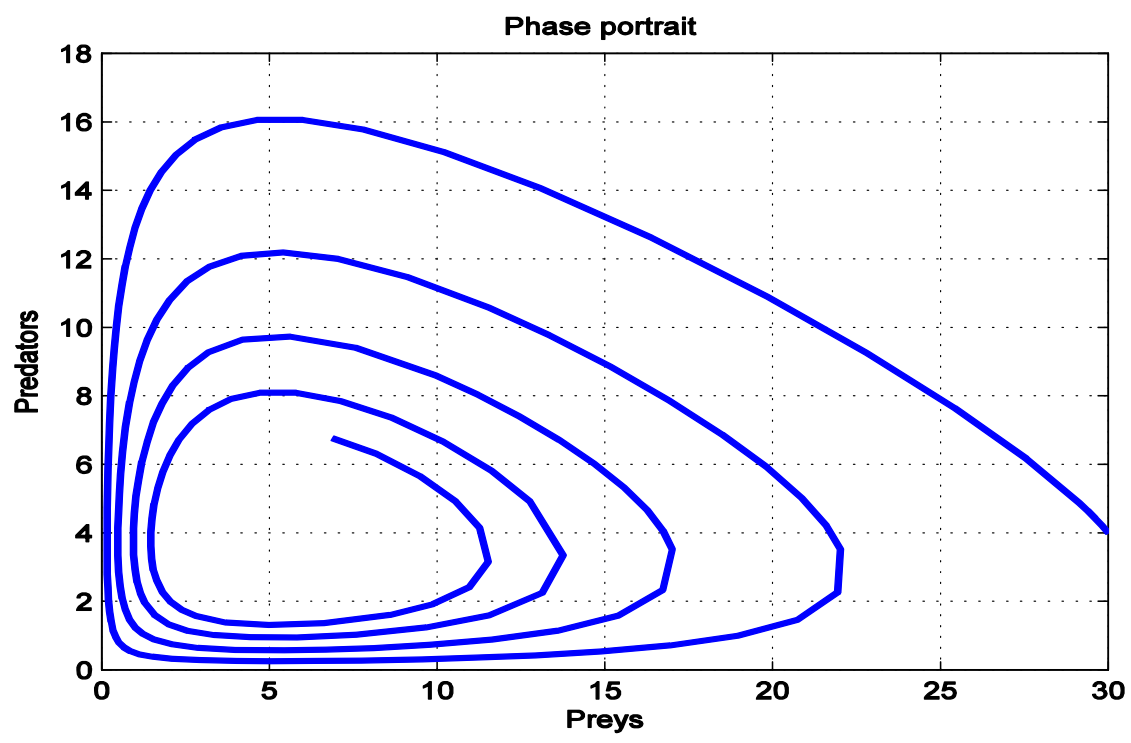

Figure 1: Global phase portrait of System of equations (3) for critical point (0, 0), (5, 3))

For the solution of system of equations (1) represents a fixed point at which both populations sustains their current, non-zero numbers and in the simplified model (2) do so indefinitely. For any positive initial populations, predator-prey populations' oscillation trajectories make periodic orbits around the point $(5,3)$. We could not calculate period exactly that why we need the numerical method to approximate solutions as functions of time $t$.

Figure 1 in the first quadrant represents the trajectories of the solutions around the fixed point $\left(N_{1}=5, N_{2}=3\right)$ and it is explained below:

- Under center: low predator implies growing of the prey population.

- Right center: high prey population implies more food and population increase.

- Over center: high predators' population eats prey and prey population decreases.

- Left Center: low prey population implies less food and predators decrease (math).

This information implies that the variation of both population species converge around 5 preys and 3 predators due to its stability. From the explanations and observations in Figure 1, it is impossible to forward to point $(0,0)$ i.e. all points go away from zero due to its instability. The vanishing of both species is not possible.

\section{Numerical simulations}

An interesting aspect about simulations is that, after the model has been validated, the researcher can change the model parameters, as well as the values used as initial conditions for the variables, and observe what happens with the results. Doing so, simulations can help researchers to make predictions about what is the response to different conditions. A larger number of hypotheses can be tested faster than their equivalent experiments, with lower costs and without exposing researchers or participants to risks. Finally, models can be used with optimization purposes. Given a scenario, which variable values can maximize or minimize an output? 


\subsection{Graphical representation}

Numerically, spatial predator-prey model represents the oscillation of both populations over time and authors used synthetic data of two interacting populations let us say for instance: wolves and rabbits in the environment containing the introductory rate of $\left(N_{1}: N_{2}=30: 4\right)$ for rabbits and wolves respectively. The growth and the decline of rabbits and wolves in fifty months are graphically observed and discussed through simulations and residuals analysis. We first substitute the estimated parameters from Table 2 in (1) and get the system of equations (3).

$$
\left\{\begin{array}{l}
\frac{d N_{1}}{d t}=0.812 N_{1}\left(1-\frac{N_{1}}{100}\right)-0.201 N_{1} N_{2}-0.032 N_{1} ; N_{1}(0)=30 \\
\frac{d N_{2}}{d t}=-0.048 N_{2}+0.1 N_{1} N_{2}-0.0008\left(N_{2}\right)^{2} ; \quad N_{2}(0)=4
\end{array}\right.
$$

Then, we solve (3) using Runge-Kutta numerical method and get the results represented by the two plots in Figure 2.
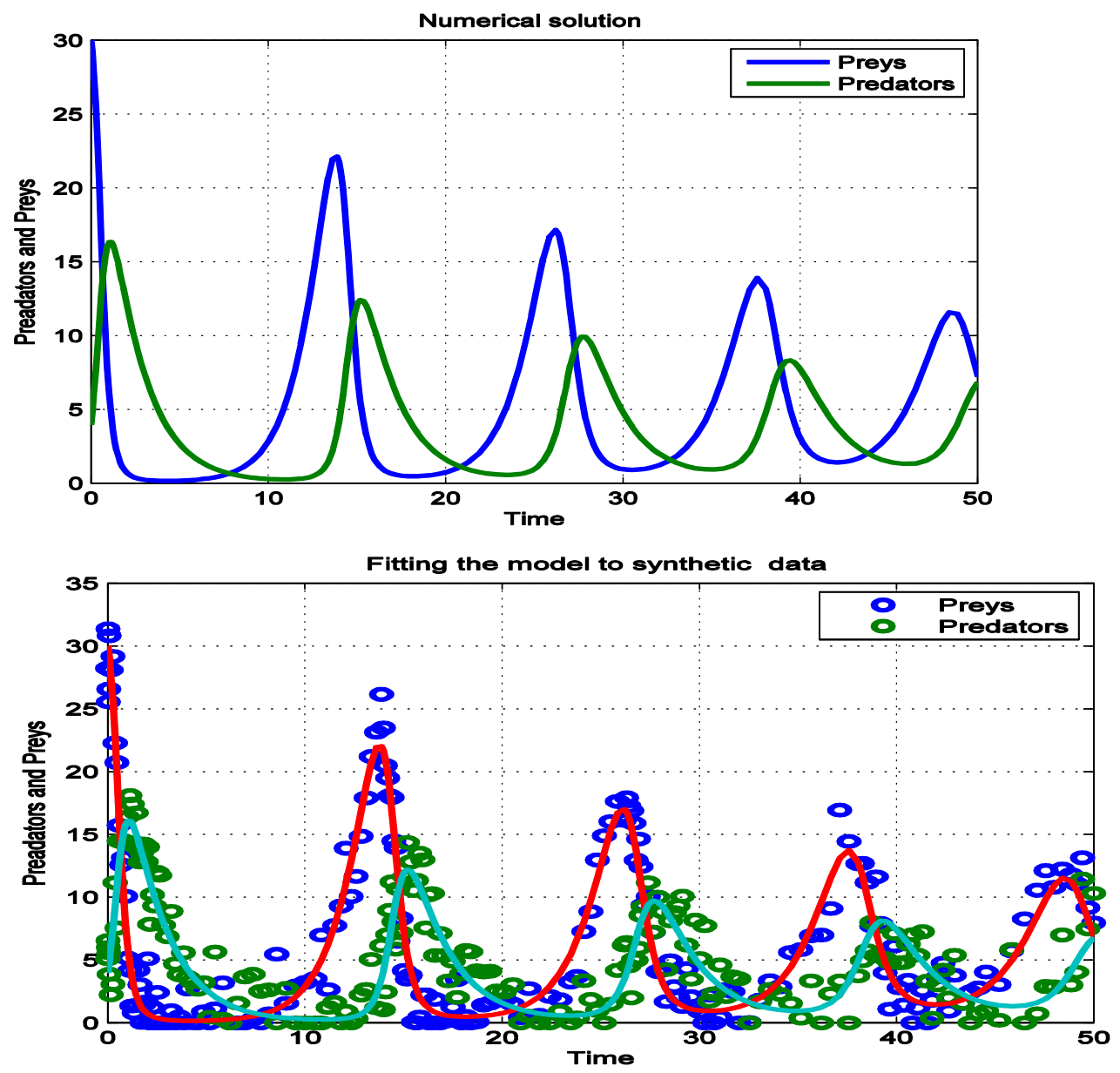

Figure 2: Numerical solutions of the system of equations (3) and the fitted models that describe the dynamism of preys and predators

From Figure 2, it is seen that predators get reproduced and increase in numbers because of their feeding while preys start to decrease. The same process is repeated over a certain time of period 
which causes the system to be periodic. It is also visible on the same figure that the ecosystem cannot be empty due to immigration and emigration. We also observe that the fitted values look like the actual values i.e. no big difference between them. This means that the actual preys and predators do not differ too much from their fitted values. This implies that there is a constant variance. Also, the expected values of residuals would be closer to zero. Therefore, according to all observations which come from the Figure 2 we should conclude that the accuracy of the model is high. The results show us that the minimum sum square of residuals denoted by $R S S$ is $R S S=2.7215 \times 10^{-3}$ and $R^{2}=0.9693$. The value of $R^{2}$ is very closer to 1 . This indicates the goodness fit of the model. This value shows that the model fits the data about $96.93 \%$. Therefore, we can conclude that the simulated data is well fitted by the spatial predator-prey model.

\subsection{Posterior distribution}

To reduce the uncertainties in parameters estimation, the MCMC method is used to produce a chain of posteriors. The Metropolis-Hastings algorithm described below is used (Ndanguza Rusatsi, 2015)

\section{Algorithm 1 Metropolis-Hastings algorithm}

1. start with arbitrary value $\theta_{0}$,

2. update from $\theta_{n}$ to $\theta_{n+1}(n=0,1 \ldots)$ by

- generating $\xi \sim q\left(\xi \backslash \theta_{n}\right)$,

- evaluate $\alpha\left(\theta_{n}, \xi\right)=\min \left(1, \frac{\pi(\xi) q\left(\xi, \theta_{n}\right)}{\pi\left(\theta_{n}\right) q\left(\theta_{n}, \xi\right)}\right)$,

- set $\theta_{n+1}=\left\{\begin{array}{c}\xi, \text { withprobability } \alpha \\ \theta_{n}, \text { Otherwise }\end{array}\right.$

The distribution $\pi(\theta)$ is often called the target distribution whereas the distribution with density $q(. \mid \theta)$ is the proposal distribution. If the symmetric proposal distribution holds; i.e. $q\left(\xi, \theta_{n}\right)=q\left(\theta_{n}, \xi\right)$, a particular case of the Metropolis-Hastings is found called Metropolis algorithm. The probability for the move is

$$
p\left(\theta_{n}, \xi\right)=q\left(\theta_{n}, \xi\right) \alpha\left(\theta_{n}, \xi\right) \text {. }
$$

The important thing to check is the detailed balance equation

$$
\pi\left(\theta_{n}\right) p\left(\theta_{n}, \xi\right)=\pi(\xi) p\left(\xi, \theta_{n}\right)
$$

which shows that $\pi$ is a stationary (invariant) distribution of the chain (CHIB \& Greenberg, 1995; Gilks, W. R., Richardson, S., and Spiegelhalter, 1996; Ndanguza Rusatsi, 2015)

$$
\int \pi\left(\theta_{n}\right) p\left(\theta_{n}, \xi\right) d \theta_{n}=\pi(\xi)
$$


Combined with the Markov chain theory, theoretically this proves that the sampling produces correct results. The sequence of iterations $\theta^{1}, \theta^{2}, \ldots$ converges to the target distribution in two steps: first, if the Markov chain is irreducible, aperiodic, and not transient, this simulated sequence has a unique stationary distribution. Second, the stationary distribution equals this target distribution and the convergence to the target distribution is proved in the same way for the Metropolis algorithm (Gilks, W. R., Richardson, S., and Spiegelhalter, 1996).

In general, the proposal distributions used in MCMC algorithms should result in well mixing of chains and in a suitable acceptance rate. Determining which proposal distribution is the best one for a particular target distribution is a very important, but also a difficult task, because it involves much trial-and-error (Ndanguza et al., 2017). The most used proposal distribution is the Gaussian distribution; however, we do not know how to obtain a suitable covariance matrix. One way to overcome this problem is to use adaptive MCMC where the proposal distribution is automatically adapted during the MCMC run (Haario, H., Saksman, E., 2001; Haario, Laine, Mira, \& Saksman, 2006).

\subsection{Diagnostics of convergence}

A convergence diagnostic procedure is a method for assessing how long to run a Markov chain in order to obtain observations from the stationary distribution of the Markov chain (Brooks, 1998). From our theory of Markov chains, we expect our chains to eventually converge to the stationary distribution, which is also our target distribution. However, there is no guarantee that our chain will converge after M draws (Brooks, 1998; Cowles \& Carlin, 1996).

There are several tests we can do, both visual and statistical, to see if the chain appears to be converged. All these tests are summarised in the work of (Cowles \& Carlin, 1996) and can be also found in (A. Gelman, J.B. Carlin, H.S. Stern, and D.B. Rubin, 1996; Brooks, 1998).

From Table 3 it is shown that the LSQ and MCMC methods are almost producing similar results.

Table 3: Identification of model parameters by LSQ

\begin{tabular}{llccc}
\hline Parameters & LSQ & Posterior mean & Median & $\begin{array}{c}\text { Standard } \\
\text { deviation }\end{array}$ \\
\hline$\alpha$ & 0.812 & 0.7998 & 0.790 & 0.0039 \\
$d_{1}$ & 0.201 & 0.0221 & 0.0220 & 0.0405 \\
$\beta$ & 0.1001 & 0.105 & 0.1051 & 0.0522 \\
$\sigma$ & 0.032 & 0.0298 & 0.0299 & 1.3599 \\
$\mu$ & 0.0008 & 0.0007 & 0.0007 & 2.7727 \\
$d_{2}$ & 0.048 & 0.051 & 0.052 & 0.0240 \\
\hline
\end{tabular}




\subsubsection{MCMC trace plot and density plots}

One of the methods for detecting lack of convergence is to examine the traces of several MCMC chains initialized with different starting values. An approach to testing the convergence is to use the MCMC trace plot and Marginal density plot. Figure 3 indicates that mixing of the samples is relatively good.
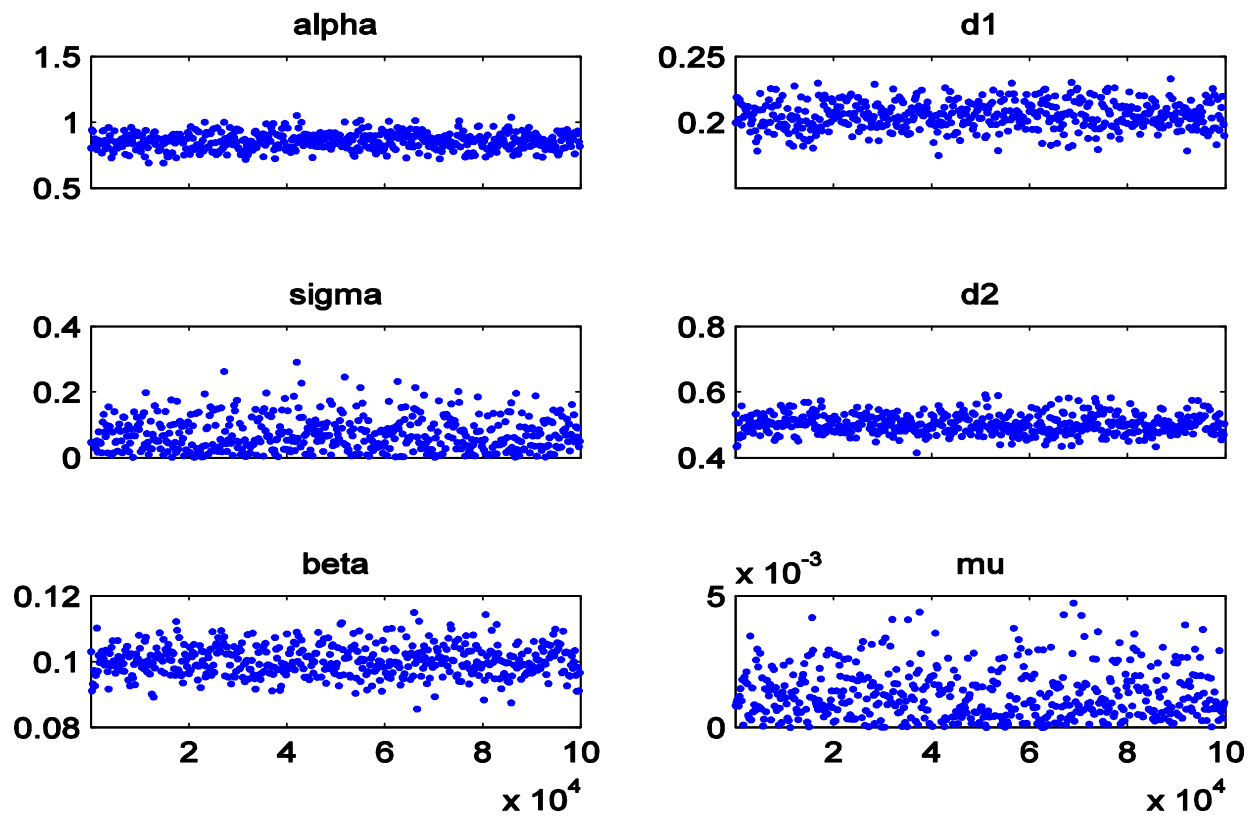

Figure 3: MCMC chains of parameters are represented as a trace plot and show the values the parameter took during the runtime of the chain. It seems that there is a perfect mixing since samples can move from one region to another in 1 step. It is the easiest way.

We may also want to visualize the marginal posterior distributions or simply marginal density plot, see Figure 4. It is the best way to use a kernel density estimate of the posterior to smooth the distributions (A. Gelman, J.B. Carlin, H.S. Stern, and D.B. Rubin, 1996; Ndanguza et al. 2017). 

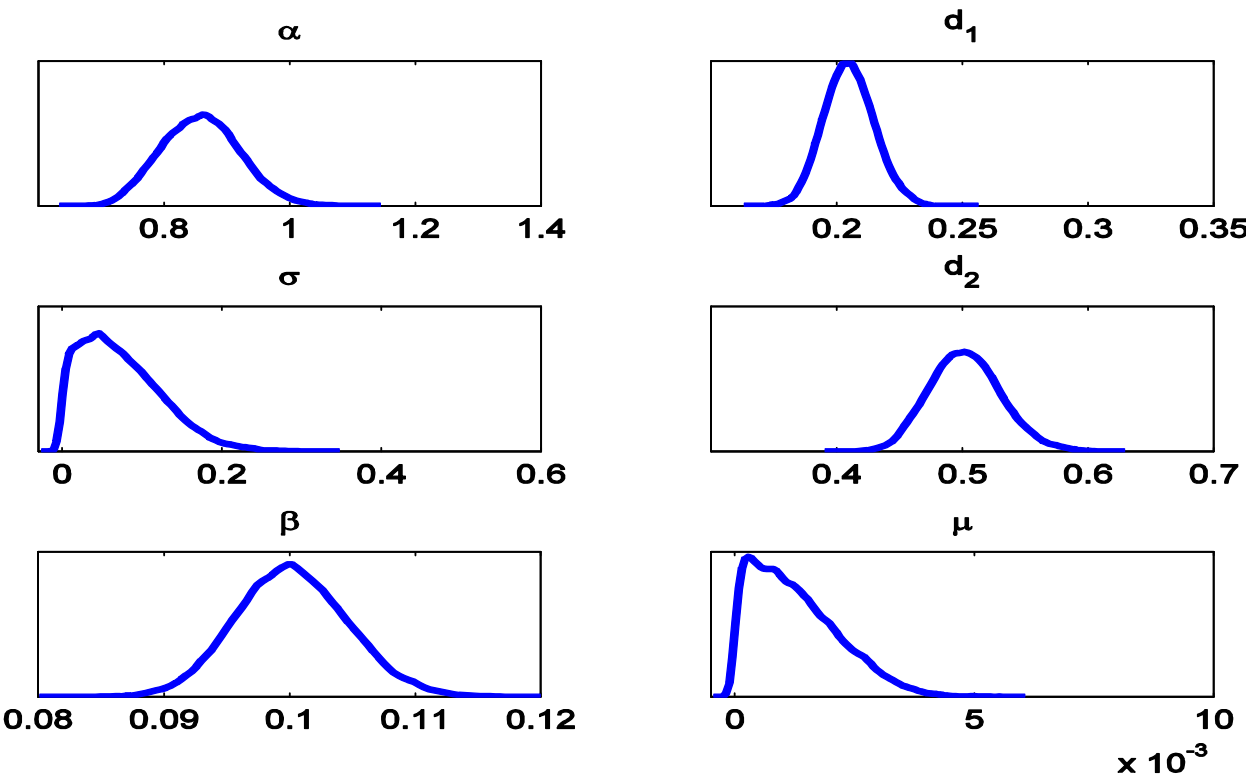

Figure 4: Kernel density plots of the six studied parameters $\left(\alpha, d_{1}, \beta, \sigma, \mu\right.$ and $\left.d_{2}\right)$. Basically, it is the (smoothed) histogram of the values in the trace-plot, i.e. the distribution of the values of the parameter in the chain. Marginal densities are an average over the values a parameter takes with all other parameters "marginalized", i.e. other parameters having any values according to their posterior probabilities. When their distribution is Gaussian, this is a positive test of convergence.

\subsubsection{Histogram plots}

Histograms shown in Figure 5 are used to plot the density of data, and often for density estimation: estimating the probability density function of the underlying variable. 

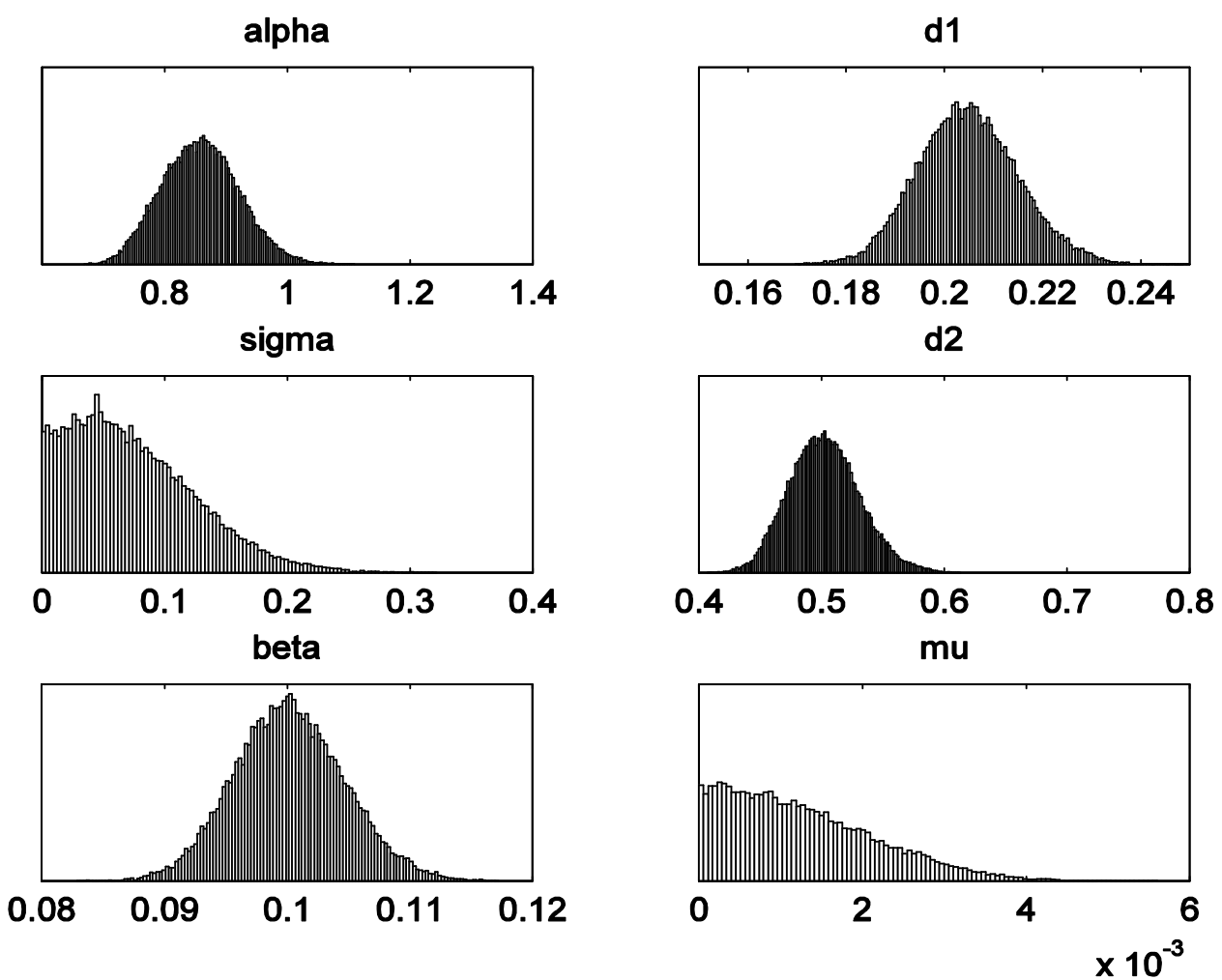

Figure 5: Histograms plot for the model parameters.

The total area of a histogram used for probability density is normalized to 1 . If the length of the intervals on the $\mathrm{x}$-axis are all 1 , then a histogram is identical to a relative frequency plot. It is seen that most of the parameters' distributions are Gaussian, which is a sign of good mixing. 


\subsubsection{Pairs}

The data is displayed as a collection of points, each having the value of one variable determining the position on the horizontal axis and the value of the other variable determining the position on the vertical axis. We plot Figure 6 showing the scatter between two by two of the parameters and check if there is a strong correlation among them.
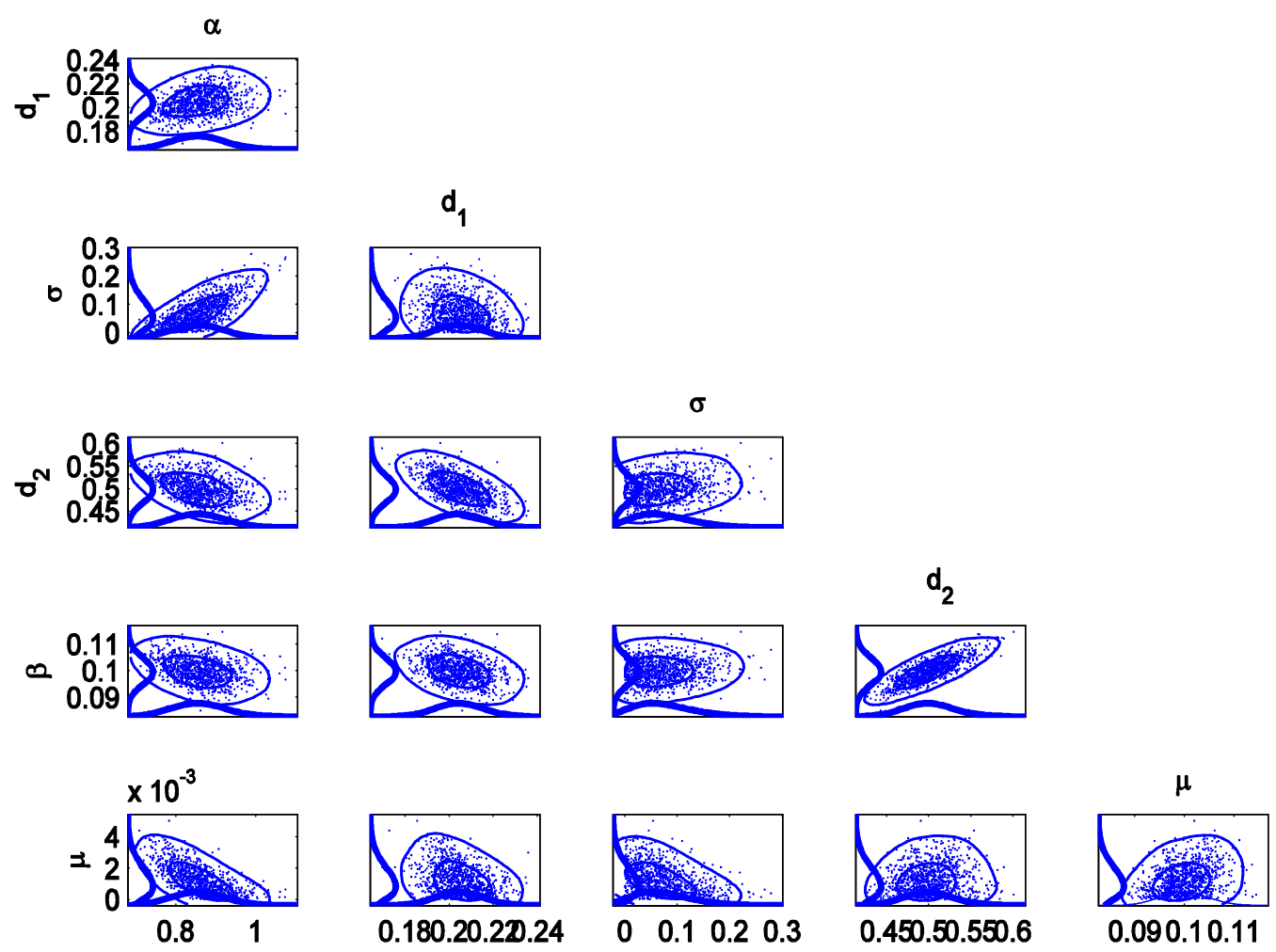

Figure 6: 2D (Together with 1D) marginal parameter posteriors plot.

High correlation is an indicator of poor mixing, so that we need a larger sample size to obtain a comparable variance. From this Figure, it is seen that there is no strong correlation among pairs of posteriors distribution which means that there is a good mixing. 


\subsubsection{Autocorrelation diagnostic}

We would expect the $\mathrm{k}^{\text {th }}$ lag autocorrelation to be smaller as $\mathrm{k}$ increases (our $1^{\text {st }}$ and $10^{\text {th }}$ draws should be less correlated than our $1^{\text {st }}$ and $2^{\text {nd }}$ draws). This is depicted in Figure 7.
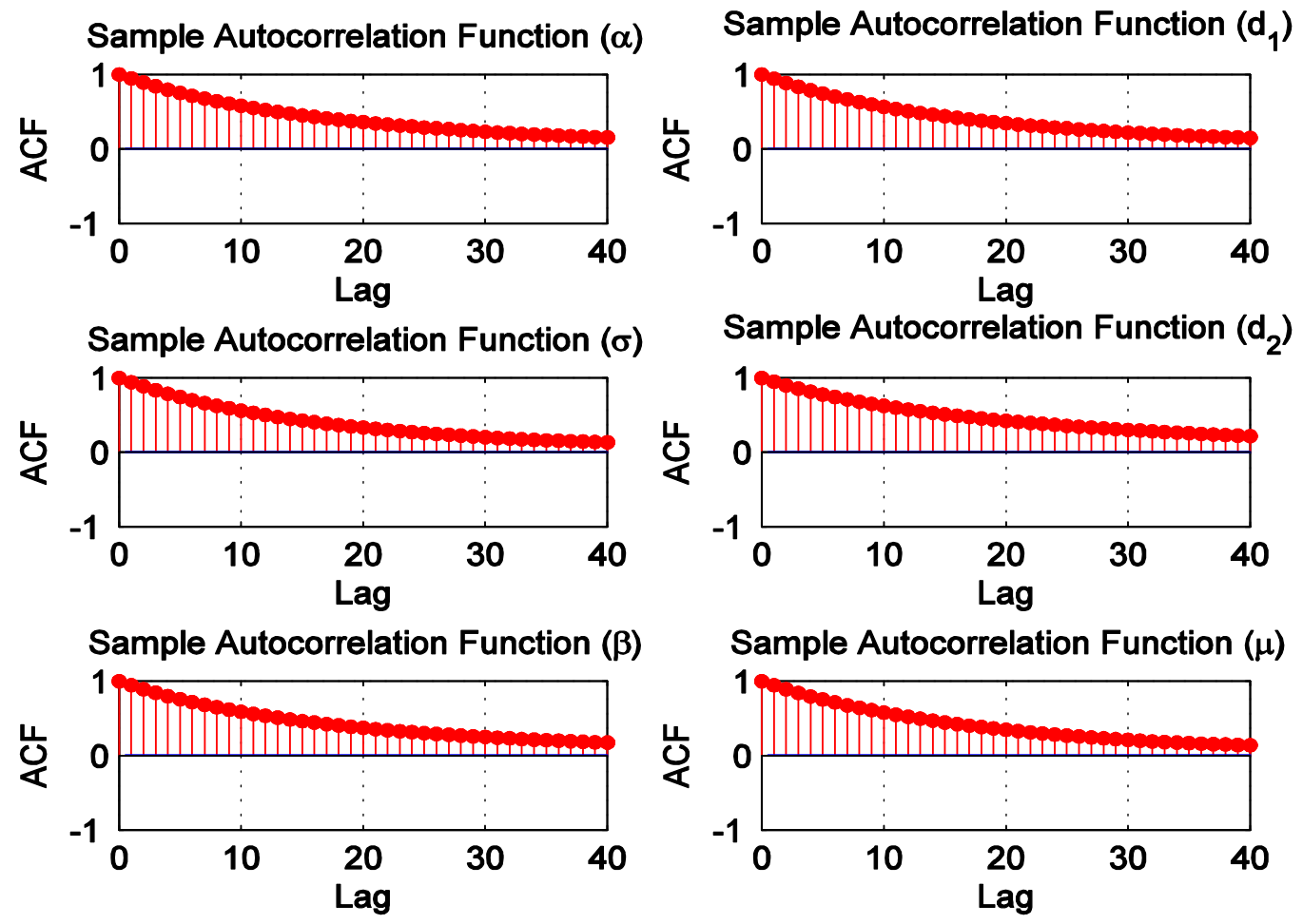

Figure 7: Estimated parameters autocorrelation functions with 40 lags.

The autocorrelation coefficients (x-axis) decay toward zero, and stabilize around zero as the number of lags (y-axis) increases. This is a sign of a good mixing. This allows users to examine the relationship among successive samples within sampled chains.

\subsubsection{MCMC predictive}

Besides the parameters distributions, we are also interested in how the uncertainty in parameters affects the model prediction. We derive the distribution for the response curves of the model; instead of one fit, we get an area where the model prediction lies with certain probability. The MCMC predictive plot is depicted in Figure 8. 

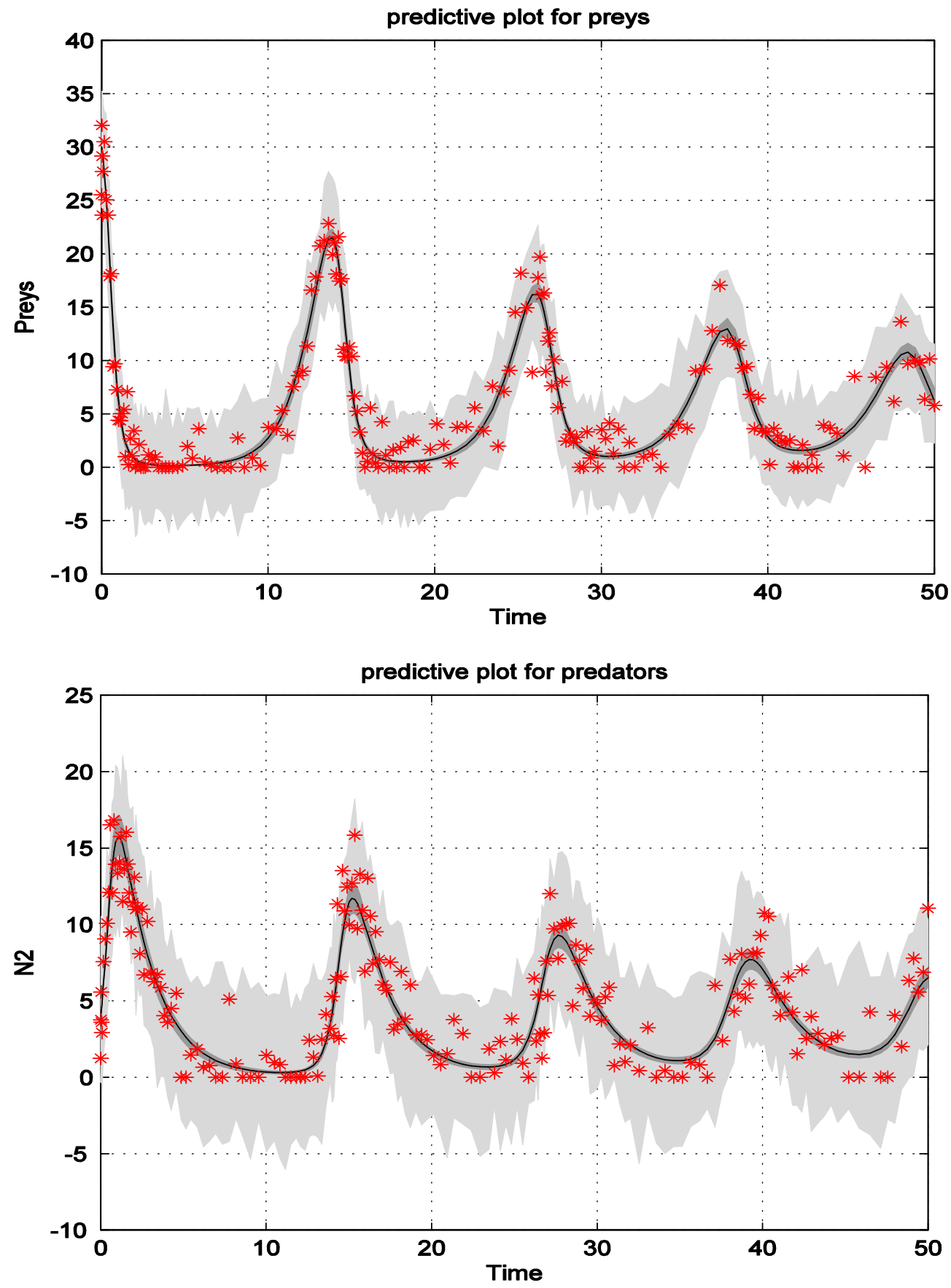

Figure 8: MCMC predictive fitting the model solution to $N_{1}(t)$ and $N_{2}(t)$ daily data. The grey areas in the plot correspond to $95 \%$ posterior regions. In the plots two kind of "areas" are plotted around the most probable response curve.

First of all, we simulate the model response with the sampled parameter values, and form a confidence interval for the response at certain points in the x-axis. This area is plotted with darker grey color and represents the area where the model prediction curve lies with a certain 
probability. Secondly, we add our estimate of the measurement error to the simulated responses to produce "noisy responses". These explanations can be summarized in Figure 9.
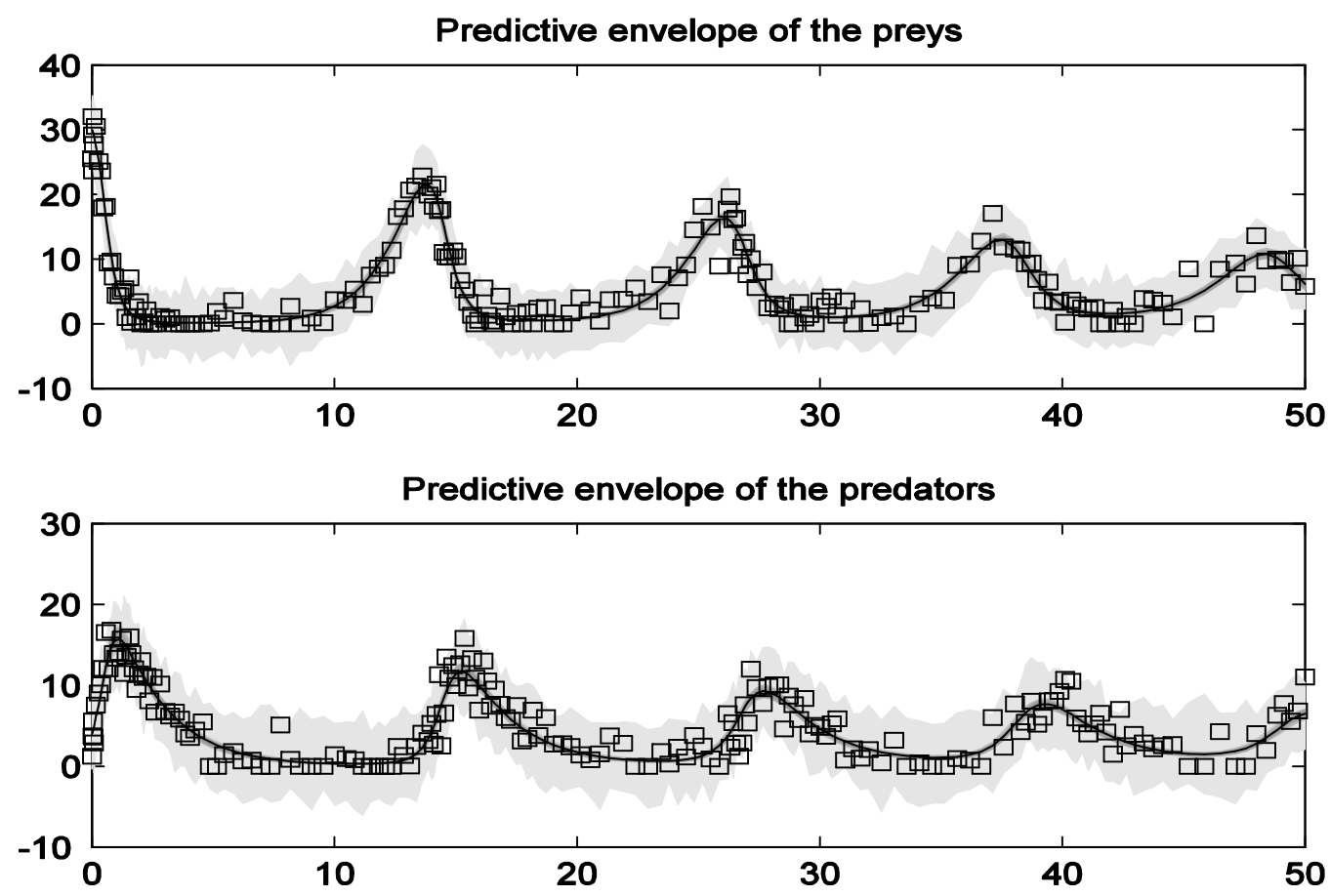

Figure 9: MCMC predictive plot

Then we similarly form a confidence interval at certain points in the x-axis for these responses, and fill the area with a lighter gray color. This represents the area from which the observations (current and forthcoming) can be found with a certain probability (Ndanguza et al., 2017). From Figure 9, one can say that uncertainties do not affect too much our formulated model and this proves the robustness of the model equations (1).

\section{Conclusion}

The Spatial predator-prey model in which the preys and predators were allowed to emigrate or immigrate into the system was established. Its parameters were estimated using least square and MCMC methods. We used regression analysis as statistical method to investigate the interaction between prey and predator. Our model was very useful in dynamical behavior and established various conditions under which the prey can exist with or without predation. The numerical analysis results indicate that the extinction of each species is impossible either absence or presence of preys or predators even though the prey and predator migrants allow. Extinction of population should only occur when the emigrants' rate of the preys exceeds their intrinsic growth rate or when prey's immigrant rate is less than death rate at which predator destroy prey. Also if the natural death of predator population is greater than their immigration rate and also if the rate at which predators increase by consuming prey does not exceed their emigrants, therefore the predators and preys would become extinct over time. The numerical analysis results show that 
the model fits the data at $96.93 \%$ and at coefficient of determination $R^{2}=0: 9693$. It was proven that both populations will continue to exist in ecosystem at a proportionality rate $N_{1}: N_{2}=5: 3$ with introductory rate $N_{1}: N_{2}=30: 4$. Otherwise there will be shrinking of the populations. Further work should aim at using the primary data for estimating the parameters of the model. The limitation of our paper is to obtain a primary data as a case study for estimating the true parameters of the model. This model is robust and should be adopted as reliable model in ecological system, like parks or fish pond for studying the evolution of different species. It can also be expanded by including other variables. The next researchers should model and analyze the spatial predator-prey system with harvesting.

\section{References}

A. Gelman; J.B. Carlin; H.S. Stern; and D.B. Rubin. (1996). Bayesian Data Analysis (Vol. 146). American Journal of Epidemiology.

Bergström, U., Englund, G., \& Leonardsson, K. (2006). Plugging Space into Predator-Prey Models: An Empirical Approach. The American Naturalist, 167(2), 246-259. https://doi.org/10.1086/499372

Boccara, N. (2010). Modeling complex systems. Springer Science \& Business Media. https://doi.org/10.1007/978-1-84882-981-7_3

Brooks, S. P. (1998). Markov chain Monte Carlo method and its application. Journal of the Royal Statistical Society Series D: The Statistician, 47(1), 69-100. https://doi.org/10.1111/14679884.00117

CHIB, S., \& Greenberg, E. (1995). Understanding the Metropolis-Hastings Algorithm. American Statistician, 49(4), 327-335. https://doi.org/10.2307/2684568

Cowles, M. K., \& Carlin, B. P. (1996). Markov Chain Monte Carlo Convergence Diagnostics: A Comparative Review. Journal of the American Statistical Association, 91(434), 883-904. https://doi.org/10.1080/01621459.1996.10476956

Cuddington, K. M., \& Yodzis, P. (2000). Diffusion-Limited Predator-Prey Dynamics in Euclidean Environments: An Allometric Individual-Based Model. Theoretical Population Biology, 58(4), 259-278. https://doi.org/10.1006/TPBI.2000.1493

Cushing, J. M., \& Levarge, S. (2005). Some Discrete Competition Models and the Principle of Competitive Exclusion. Difference Equations and Discrete Dynamical Systems, Proceedigns of the Ninth International Conference, 283-302. https://doi.org/10.1080/10236190410001652739

Gilks, W. R., Richardson, S., and Spiegelhalter, D. J. (1996). Markov chain Monte Carlo in Practice. Taylor \& Franics group.

Haario, H., Saksman, E., and T. (2001). An adaptive Metropolis algorithm, 7(12), 223-242. Retrieved from https://scholar.google.com/

Haario, H., Laine, M., Mira, A., \& Saksman, E. (2006). DRAM: Efficient adaptive MCMC. Statistics and Computing, 16(4), 339-354. https://doi.org/10.1007/s11222-006-9438-0

Jang, S. J., \& Baglama, J. (2002). A nutrient-prey-predator model with intratrophic predation. Applied Mathematics and Computation, 129(2-3), 517-536. https://doi.org/10.1016/S00963003(01)00060-1

KOROBEINIKOV, A. and WAKE, G. . (1999). Global properties of. Journal of Applied Mathematics, 3(2), 155-162. https://doi.org/10.1216/RMJ-1980-10-4-665 
Liu, P. P. (2010). An analysis of a predator-prey model with both diffusion and migration. Mathematical and Computer Modelling, 51(9-10), 1064-1070. https://doi.org/10.1016/j.mcm.2009.12.010

Lugo, C. A., \& McKane, A. J. (2008). Quasicycles in a spatial predator-prey model. Physical Review E - Statistical, Nonlinear, and Soft Matter Physics, 78(5). https://doi.org/10.1103/PhysRevE.78.051911

Ndanguza, D., Mbalawata, I. S., Haario, H., \& Tchuenche, J. M. (2017). Analysis of bias in an Ebola epidemic model by extended Kalman filter approach. Mathematics and Computers in Simulation, 142, 113-129. https://doi.org/10.1016/J.MATCOM.2017.05.005

Ndanguza Rusatsi, D. (2015). Bayesian Analysis of SEIR epidemic models. Retrieved from https://www.lut.fi/web/en/news/-/asset_publisher/1Gh4SAywhcPu/content/bayesiananalysis-of-seir-epidemic-models

Pal, D., Mahapatra, G. S., \& Samanta, G. P. (2015). Stability and bionomic analysis of fuzzy parameter based prey-predator harvesting model using UFM. Nonlinear Dynamics, 79(3), 1939-1955. https://doi.org/10.1007/s11071-014-1784-4

Vivek, S. K. K. (2015). Analysis of an Eco-Epidemiological Model with Migrating and Refuging Prey. Springer Proceedings in Mathematics and Statistics, 143. https://doi.org/10.1007/97881-322-2485-3

Wilson, E. O., and W. H. B. (1971). A primer of population biology. Sinauer Associates Sunderland, MA, 3, 607-608. https://doi.org/10.2307/1351023 